\section{THU0205 THE EFFECT OF CERTOLIZUMAB DRUG CONCENTRATION AND ANTI-DRUG ANTIBODIES ON TNF NEUTRALISATION}

L.C. Berkhout ${ }^{1}$, E.H. Vogelzang ${ }^{2}$, M.H. Hart ${ }^{1}$, N.I. Derksen ${ }^{1}$, R. Wieringa ${ }^{3}$, W. A. van Leeuwen ${ }^{3}$, C.L. Krieckaert ${ }^{2}$, A. de Vries ${ }^{3}$, M.T. Nurmohamed ${ }^{2,4}$, G. Wolbink ${ }^{1,2}, \mathrm{~T}$. Rispens ${ }^{1} .{ }^{1}$ Department of Immunopathology, Sanquin Research; ${ }^{2}$ Amsterdam Rheumatology and immunology Center, Reade; ${ }^{3}$ Biologics Lab, Sanquin Diagnostic Services; ${ }^{4}$ Amsterdam Rheumatology and immunology Center, VU University Medical Center, Amsterdam, Netherlands

Background: Although tumour necrosis factor- (TNF) inhibitors have proven to be a successful treatment option for patients with rheumatoid arthritis (RA), TNFinhibitors, including certolizumab, elicit an immunogenic response leading to the formation of anti-drug antibodies (ADAs) (reported range $\sim 5 \%-39 \%$ of the patients).

Objectives: We sought to investigate the relationship between certolizumab concentrations, ADAs, and the effective TNF neutralising capacity in sera of RA patients. TNF neutralising capacity of certolizumab was compared to the neutralising capacity of adalimumab.

Methods: Blood was collected of 35 consecutive certolizumab-treated RA patients at baseline and 4, 16, 28 and 52 weeks after treatment initiation. Certolizumab and ADA levels were quantified using a certolizumab bridging enzymelinked immunosorbent assay (ELISA) and a drug-tolerant radioimmunoassay (RIA), respectively. TNF neutralisation of certolizumab and adalimumab in patient sera, in presence or absence of ADAs, was analysed using the TNF-sensitive WEHI bioassay.

Results: Despite a high incidence of ADAs during one year of follow-up (69\%;24/ 35 patients), certolizumab levels of $\sim>10 \mu \mathrm{g} / \mathrm{mL}$ were measured in most patients (Spearman's $r=-0.7155, p<0.0001$ ( $n=118)$; figure 1A). Furthermore, TNF neutralisation, expressed by EC50 values, was highly correlated with certolizumab serum concentration, while there was no association with ADAs (Pearson $r=0.9087, p<0.0001(n=12)$ and Pearson $r=-0.4712, p=0.1220(n=12)$; figure 1B and $C$, respectively. Grey lines indicate log-log linear fit, weight by $1 / Y^{\wedge} 2$ ). Similar results were obtained for adalimumab, although TNF neutralisation by adalimumab was less potent; the relative in vitro neutralising potency was 43 times higher for certolizumab compared to the neutralising potency of adalimumab.
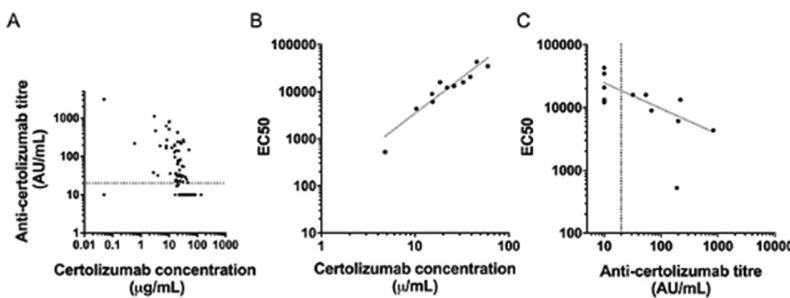

Conclusions: Our study shows that certolizumab is highly immunogenic. In most cases where ADAs are detected, certolizumab is also present in high amounts, and can still potently neutralise TNF. Furthermore, TNF neutralisation is highly correlated with certolizumab concentrations. Therefore, measurement of certolizumab concentrations is the relevant parameter to assess clinically relevant immunogenicity.

Disclosure of Interest: L. Berkhout: None declared, E. Vogelzang: None declared, M. Hart: None declared, N. Derksen: None declared, R. Wieringa: None declared, W. van Leeuwen: None declared, C. Krieckaert Speakers bureau: Pfizer, A. de Vries: None declared, M. Nurmohamed Consultant for: Abbott, Roche, Pfizer, MSD, UCB, SOBI, BMS, Speakers bureau: Abbott, Roche, Pfizer, G. Wolbink Grant/research support from: Pfizer, Speakers bureau: Pfizer, UCB, AbbVie, Biogen, BMS, T. Rispens Grant/research support from: Genmab, Speakers bureau: Pfizer, AbbVie, Regeneron

DOI: 10.1136/annrheumdis-2018-eular.4399

\section{THU0206 \\ ADD-ON SHORT-COURSE TOCILIZUMAB ACCELERATES DOSE TAPERING OF GLUCOCORTICOIDS IN RHEUMATOID ARTHRITIS: RESULTS FROM A CHINESE PROSPECTIVE COHORT STUDY}

J.-W. Wang ${ }^{1}$, Y.-Q. Mo ${ }^{1}$, X.-Y. Wang ${ }^{2}$, L.-F. Chen ${ }^{1}$, J.-D. Ma ${ }^{1}$, J.-Z. Lin ${ }^{1}$, D.H. Zheng ${ }^{1}$, L. Dai'. 'Department of Rheumatology and Immunology, Sun Yat-sen Memorial Hospital, Sun Yat-sen University, Guangzhou; ${ }^{2}$ Department of Rheumatology and Immunology, The First Affliated Hospital of Zhengzhou University, Zhengzhou, China

Background: In real-world clinical practice, self-paid and expensive price limit the application of bDMARDs and only a few patients especially in developing countries could afford long-term use. Only $9.1 \%>11 \%$ of Chinese rheumatoid arthritis (RA) patients were treated with bDMARDs with mean course no more than 6 months. Such short course of bDMARDs had always been raised doubt about their efficacy and benefit for rare reported evidence.

Objectives: To explore the efficacy of additional short-course of Tocilizumab (TCZ) combined with csDMARDs in real-world RA management.

Methods: Consecutive patients with active RA (DAS28-ESR>2.6) who had completed 6 month follow-up were retrospectively recruited from a prospective RA cohort $(n=582)$. All patients were treated according to the treat-to-target strategy and patient's willingness especially biologics use. RA patients who finished at least 3 infusions of TCZ (8 mg/Kg/4 weeks) were included as add-on TCZ group, and matched RA patients without any bDMARDs by age, sex and disease activity at baseline with the ratio of $1: 1$ were included as csDMARDs group. Clinical data were collected according to the 2017 EULAR recommendation at baseline and regular visits at week 4,12 and 24 .

Results: (1) The baseline characteristics of 101 paired RA patients showed no significant difference except for lower csDMARDs-naïve percentage between two groups (table 1). (2) During 24 week follow up, there were significantly higher percentage of patients in add-on TCZ group achieving therapeutic target (DAS28ESR $<3.2$, at Week $4: 59 \%$ vs. $39 \%, p=0.005$; at Week $12: 71 \%$ vs. $52 \%, p=0.006$, figure 1) or remission (DAS28-ESR<2.6, at Week 4: 46\% vs. $16 \%, p<0.001$; at Week $12: 53 \%$ vs. $28 \%, p<0.001$ ) than those in csDMARDs group. Furthermore, there were significantly higher percentage of patients in add-on TCZ group achieving deep remission (DAS28-ESR $\leq 1.98$, at Week $4: 16 \%$ vs. $5 \%, p=0.015$ at Week $12: 28 \%$ vs. $9 \%, p=0.001$; at Week $24: 20 \%$ vs. $9 \%, p=0.046$ ). (3) There were $76 \%$ RA patients in add-on TCZ group with glucocorticoids (GC) therapy which is significantly lower than that in csDMARDs group (92\%, $p=0.002)$. Among patients with GC therapy, the GC dosage per day was tapered more rapidly at each visit and the cumulative dose at week 24 was significantly lower in add-on TCZ group than that in csDMARDs group ( $826 \pm 616$ vs. $1128 \pm 519 \mathrm{mg}, \mathrm{p}<0.001)$.

Table 1. Baseline characteristics of RA patients in add-on TCZ group or csDMARDs group

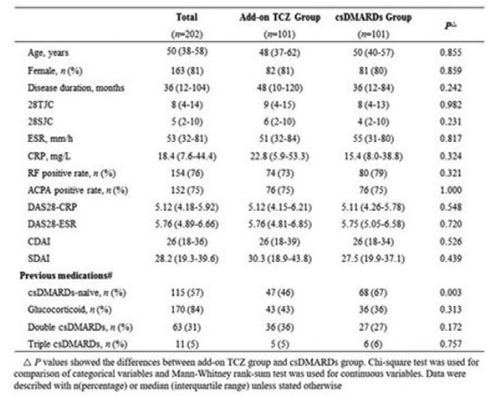

Conclusions: Add-on short-course TCZ may be an alternative induction strategy for RA patients in developing countries which can quickly achieve target and accelerate dose tapering of $\mathrm{GC}$.

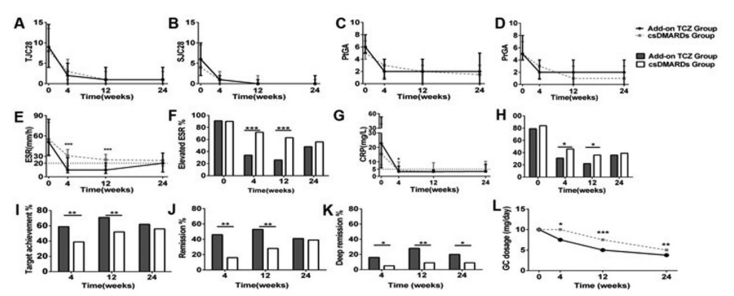

Abstract THU0206 - Figure 1. Dynamic changes of therapy indexes between add-on TCZ group and csDMARDs group. (A-H) Comparison of disease activity indexes at baseline and week 4, 12 and 24. (I-K) Comparison of therapeutic effect. (L) Comparison of glucocorticoid dosage per day. Therapeutic target, remission and deep remission were defined as DAS28ESR $<3.2,<2.6$ and $\leq 1.98$, respectively. ${ }^{*} P<0.05 .{ }^{* \star} P<0.01 .{ }^{\star \star \star} P<0.001$. 
Acknowledgements: This work was supported by National Natural Science Foundation of China (grant no. 81471597, 81671612 and 8160147) and Guangdong Natural Science Foundation (grant no. 2016A030313307 and 2017A030313576)

Disclosure of Interest: None declared

DOI: 10.1136/annrheumdis-2018-eular.5130

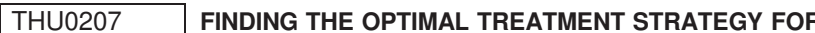 DISEASE ACTIVITY-GUIDED DOSE REDUCTION OF ADALIMUMAB AND ETANERCEPT IN RHEUMATOID ARTHRITIS: A MODELLING STUDY}

L.M. Verhoef ${ }^{1}$, D.P. Bos ${ }^{1}$, C.H. van den Ende ${ }^{1,2}$, F.H. van den Hoogen ${ }^{1,2}$, B. Fautrel ${ }^{3}$, M.E. Hulscher ${ }^{4}$, W. Kievit ${ }^{5}$, A.A. den Broeder ${ }^{1,2} .{ }^{1}$ Rheumatology, Sint Maartenskliniek; ${ }^{2}$ Rheumatology, Radboudumc, Nijmegen, Netherlands; ${ }^{3}$ Rheumatology, Pitié-Salpêtrière Hospital, Paris, France; ${ }^{4} I Q$ healthcare, RIHS; ${ }^{5}$ Health Evidence, RIHS, Radboudumc, Nijmegen, Netherlands

Background: Several studies have shown that disease activity-guided dose reduction without deterioration of disease activity is possible, while saving costs in patients with stable and low disease activity. $\left({ }^{1}\right.$ Despite these positive results, questions remain on the optimal tapering strategy. Different strategies are conceivable, with varying results regarding the balance between number of flares, utilities and costs.

Objectives: The objective of this study was to investigate the most cost-effective TNFi dose reduction strategy for RA patients using a modelling design.

Methods: In a cost-utility analysis using Markov modelling based on data from the DRESS study, STRASS study, and the RA Nijmegen cohort, the following strategies were tested against continuation: 1. Four-step DRESS tapering (figure $1: 100 \%-67 \%-50 \%-0 \%) ; 2$. Tapering with an extra dosage step of $33 \%$; 3. Tapering without withdrawal; 4 . Use of a stricter flare criterion (DAS >2.6); and 5 . Use of a predictor (biomarker: $80 \%$ specific, $80 \%$ sensitive, $€ 100$ per test) for successful tapering. Scenario analyses with $30 \%$ and $50 \%$ drug price discount and no discounting were executed. Also, it was examined how well a biomarker should be able to predict to become cost-effective compared to the other strategies.

Results: All examined tapering strategies were found to be cost saving but yielded more short-lived flares compared to continuation (table 1). The change in utilities was minimal and not clinically relevant. Strategy 1 was cost-effective compared to all other strategies (highest incremental Net Monetary Benefit (iNMB)). However, there was a large overlap in credible intervals, especially between strategy 1 and 2 . Scenario analyses showed that $50 \%$ reduction of drug prices would result in the highest iNMB for strategy 2 . A biomarker only becomes cost-effective when it has a sensitivity and specificity of at least $86 \%$.

Data is mean values per patient over 1.5 years (95\% credible intervals). * Stricter flare criterion (DAS28 >2.6). ${ }^{* *} \mathrm{NMB}=\mathrm{QALYS}$ * WTP/WTA - costs; iNMBs are given at a WTP/WTA of $€ 80000$

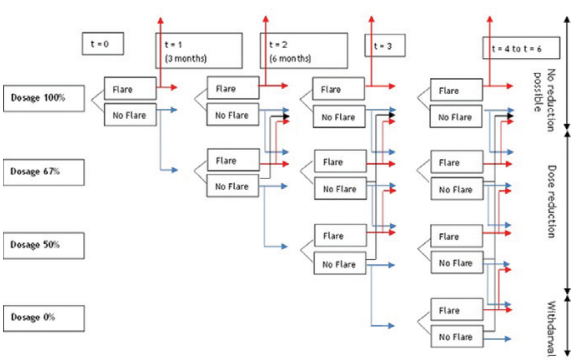

Conclusions: All dose reduction strategies dominated the continuation strategy regarding cost-effectiveness. Because our study showed a comparable iNMB for tapering in four or five steps, we recommend a choice between these strategies, based on shared decision making.

\section{REFERENCE:}

[1] Nam JL, et al. Ann Rheum Dis 2017 Jun;76(6):1113-1136.

Disclosure of Interest: L. Verhoef: None declared, D. Bos: None declared, C. van den Ende: None declared, F. van den Hoogen Consultant for: Biogen, Celltrion and Mundipharma, B. Fautrel: None declared, M. Hulscher: None declared, W. Kievit: None declared, A. den Broeder: None declared

DOI: 10.1136/annrheumdis-2018-eular.2604

\section{THU0208 EFFECTIVENESS AND SAFETY OF CERTOLIZUMAB PEGOL IN RHEUMATOID ARTHRITISPATIENTS AFTER FAILURE TO ANTITNF OR NAÏVE PATIENTS IN REAL WORLD SETTING. ONE YEAR FOLLOW-UP EXPERIENCE}

M. Fernández Prada ${ }^{1}$, V. Torrente-Segarra ${ }^{2}$, R. Expósito Molinero ${ }^{3}$, N.P. Garrido Puñal $^{4}$, A. Sánchez-Andrade Fernández ${ }^{5}$, J.R. Lamúa ${ }^{6}$, A. Olivé ${ }^{7}$, J.V. Tovar ${ }^{8}$, on behalf of RENACER Syudy Group. ${ }^{1}$ Reumatologia, Hospital Universitario Guadalajara, LAS ROZAS. MADRID; ${ }^{2}$ Reumatologia, Hospital General HospitaletMoisès Broggi, Hospitalet Llobregat, ${ }^{3}$ Reumatologia, Hospital Comarcal de Laredo, Laredo; ${ }^{4}$ Reumatologia, Hospital Universitario Virgen del Rocío, Sevilla;

${ }^{5}$ Reumatologia, Complejo Hospital Universitario Lucus Augusti, Lugo;

${ }^{6}$ Reumatologia, Hospital Universitario del Henares, Coslada; ${ }^{7}$ Reumatologia, Hospital Germans Trias i Pujol, Badalona; ${ }^{8}$ Reumatologia, Hospital General Universitario de Elche, Elche, Spain

Background: Between $30 \%-40 \%$ of patients with rheumatoid arthritis (RA) discontinue at their first line biological therapy due to inadequate response or adverse events. There is currently a great controversy about using a second antiTNF, after failure to the first one, or a therapeutic target change, due to the availability of a wide pharmacological arsenal for patients with RA

Objectives: The objective of this study is to determine the effectiveness and safety during one year of Certolizumab pegol (CZP) in patients with RA in second line (after failed to a previous antiTNF) and compare it with those who received this therapy as first line (bio-naïve).

Methods: National, observational, prospective, longitudinal and multicenter study in patients with RA who received CZP as the first line of biological treatment or failure to a previous anti-TNF for lack of efficacy, during one year of follow-up. Demographic and clinical variables were collected (gender, age, disease duration, TJC SJC, ESR, CRP, DAS28, HAQ), at baseline visit, 3 and 12 months of treatment. As response variables, DAS28 remission, low disease activity by DAS28 (LDA) and EULAR response, were used. The Student $t$ test and the Mann-Whitney $U$ test were used for the statistical analysis and a survival analysis (Kaplan-Meier) was performed

Results: A total of 360 patients were included, of which $272(75.6 \%)$ were bionaïve and $88(24.4 \%)$ previous anti-TNF failure. Baseline characteristics of both groups are shown in table 1. Clinical variables of effectiveness at 3 and 12 months are summarised in table 2. Statistically significant differences in DAS28, HAQ and $\%$ patients with corticosteroids were observed in both groups at year of treatment comparing with baseline, even though bio-naïve group had a statistically lower DAS28 and a higher percentage response than the antiTNF failure group. Accord ing to Kaplan-Meier analysis, similar retention rates were observed independently if CZP were used as first $(77.2 \%)$ or second line $(73.9 \%)$. No differences were found in terms of safety at one year of treatment between both groups.

Conclusions: This study, derived from the experience of national clinical practice with CZP, demonstrates the effectiveness and safety of this drug in patients with RA regardless of its use in the first line or after failure to a previous antiTNF, with better response scores in bio-naïve patients. No differences were observed in the retention rate in both groups.

\begin{tabular}{|c|c|c|c|c|c|c|}
\hline Outcome measure & $\begin{array}{l}\text { 0. Continuation } \\
\text { (comparator) }\end{array}$ & $\begin{array}{l}\text { 1. DRESS strategy (four-step } \\
\text { tapering) }\end{array}$ & $\begin{array}{l}\text { 2. Five-step } \\
\text { tapering }\end{array}$ & $\begin{array}{c}\text { 3. No } \\
\text { withdrawal }\end{array}$ & $\begin{array}{l}\text { 4. Stricter flare } \\
\text { criterion }\end{array}$ & 5. Predictor \\
\hline Costs $(€)$ & $\begin{array}{c}21071 \\
(20,563-21,383\end{array}$ & $\begin{array}{c}13198 \\
\left({ }^{12,469-13,988}\right.\end{array}$ & $\begin{array}{c}13794 \\
(13,150-14,496\end{array}$ & $\begin{array}{c}14266 \\
\left({ }^{13,685-14,856}\right.\end{array}$ & $\begin{array}{c}15943 \\
\left({ }^{15,115-16,793}\right.\end{array}$ & $\begin{array}{c}14327 \\
\left({ }^{13,281-15,359}\right.\end{array}$ \\
\hline QALYs & $\begin{array}{c}1.182 \\
(1.165-1.199)\end{array}$ & $\begin{array}{c}1.177 \\
(1.160-1.193)\end{array}$ & $\begin{array}{c}1.181 \\
(1.165-1.197)\end{array}$ & $\begin{array}{c}1.182 \\
(1.166-1.198)\end{array}$ & $\begin{array}{c}1.189 \\
(1.173-1.205)\end{array}$ & $\begin{array}{c}1.185 \\
(1.168- \\
1.200)\end{array}$ \\
\hline $\begin{array}{l}\text { Mean number of short-lived } \\
\text { flares }\end{array}$ & $\begin{array}{c}0.53 \\
(0.35-0.73)\end{array}$ & $\begin{array}{c}0.97 \\
(0.83-1.12)\end{array}$ & $\begin{array}{c}0.74 \\
(0.58-0.93)\end{array}$ & $\begin{array}{c}0.69 \\
(0.52-0.87)\end{array}$ & $\begin{array}{c}2.08^{*} \\
(1.80-2.41)\end{array}$ & $\begin{array}{c}0.55 \\
(0.53-0.56)\end{array}$ \\
\hline iNMB $^{\star \star}$ & - & $\begin{array}{c}7434 \\
(6,514-8,302\end{array}$ & $\begin{array}{c}7176 \\
(6,307-8,000\end{array}$ & $\begin{array}{c}6798 \\
(5,977-7,539\end{array}$ & $\begin{array}{c}5650 \\
(3,598-7,625\end{array}$ & $\begin{array}{c}6938 \\
(5,863-8,040\end{array}$ \\
\hline
\end{tabular}

